\title{
Neighbourhood environment and depressive symptoms among the elderly in Hong Kong and Singapore
}

\author{
Winnie W. Y. Lam ${ }^{1}$, Becky P. Y. Loo ${ }^{*}$ (1) and Rathi Mahendran²
}

\begin{abstract}
Background: Geriatric depression is a growing public health issue worldwide. This study aims at identifying the relevant neighbourhood attributes, separate from the individual-level characteristics, that are related to the onset of depressive disorders among the geriatric population.

Methods: This study adopts a structural equation modelling (SEM) approach to understand the effect of the neighbourhood environment on geriatric depression, as identified by data collected from community-dwelling elderly living in Hong Kong and Singapore. Using network buffers as the unit of analysis, different features of the neighbourhood environment are captured and analysed. SEM also examines the strength and direction of the relationships using different parameters at both the individual and neighbourhood levels, as well as the prevalence of depressive symptoms among the elderly.

Results: The total sample size is 347, with 173 and 174 elderly people in Hong Kong and Singapore respectively. The results show that in addition to one's physical health status, both objective and subjective neighbourhood factors including the size of parks, land use mix, walkability, and connectivity are all statistically significant influential factors in geriatric depression. In particular, enhancing walkability and providing more parks at the neighbourhood level can bring mental health benefits.

Conclusions: Public health policy initiatives aimed at tackling geriatric depression can be achieved by adopting a holistic and integrative approach to better prepare the neighbourhood environment in an ageing society.
\end{abstract}

Keywords: Depressive symptoms, Elderly, Neighbourhood environment, Hong kong, Singapore

\section{Introduction}

Depression in later life is an important public health issue due to the increased risk of morbidity and suicide, as well as decreased physical, social, and cognitive functioning [5]. In two of the most rapidly ageing cities in Asia, elderly depression is one of the most common psychiatric disorders. In Hong Kong, the prevalence rate is 13.7\% for females and $8.9 \%$ for males; while in Singapore it is

${ }^{*}$ Correspondence: bpyloo@hku.hk

${ }^{1}$ Department of Geography, The University of Hong Kong, Pokfulam Road, Hong Kong, China

Full list of author information is available at the end of the article estimated at $5.5 \%[20,44]$. Therefore, to be effective and relevant, age-related policies and programmes in Asian cities need to keep pace with the rapidly ageing population [37]. In particular, the identification of factors that exacerbate or alleviate depression have important implications in promoting healthy ageing-in-place, a concept that allows the elderly to live in their existing communities with familiar family and/or social support, rather than to move to institutionalized homes for the elderly [32].

In cities, multiple factors contribute to the risk of depression ranging from genetic vulnerabilities, neurobiological changes, and environmental factors such

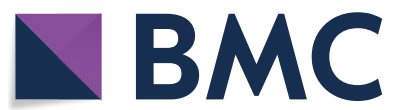

(c) The Author(s) 2020. This article is licensed under a Creative Commons Attribution 4.0 International License, which permits use, sharing, adaptation, distribution and reproduction in any medium or format, as long as you give appropriate credit to the original author(s) and the source, provide a link to the Creative Commons licence, and indicate if changes were made. The images or other third party material in this article are included in the article's Creative Commons licence, unless indicated otherwise in a credit line to the material. If material is not included in the article's Creative Commons licence and your intended use is not permitted by statutory regulation or exceeds the permitted use, you will need to obtain permission directly from the copyright holder. To view a copy of this licence, visit http://creativeco mmons.org/licenses/by/4.0/. The Creative Commons Public Domain Dedication waiver (http://creativecommons.org/publicdomain/ zero/1.0/) applies to the data made available in this article, unless otherwise stated in a credit line to the data. 
as social and psychological issues, and often the neighbourhood environment [47]. For governments, a holistic ageing policy that considers not only individual demographic, medical, and socio-economic factors but also their neighbourhood environment is needed to safeguard the physical and mental health of the elderly. In particular, the integrative conceptual framework developed by Billings and Moos [4] suggests that personal resources interact with environmental resources such as physical and architectural features of community settings which could lead to environmental stressors and affect appraisal and coping responses to cause depression. This research focusing on the neighbourhood environment and elderly depressive symptoms is grounded in this theoretical background.

Figure 1 is a schematic diagram of the integrative framework. A recent review summarises that neighbourhood environmental resources including high walkability, connectivity, and accessibility are expected to contribute to the elderly's physical and mental health, and hence their quality of life [33]. The elderly tend to spend more time in their neighbourhood environment because their activities are significantly curtailed by physical decline and frailty, retirement, reduced mobility, decreased access to transport, shrinking social support and networks, and cognitive decline [12]. Hence, satisfaction with the availability and proximity of key resources, such as retail and healthcare services, is also found to reduce depressive symptoms [24]. Good connectivity, while reducing trip distance between locations, enhances walkability and encourages outdoor mobility in older adults [42]. Similarly, open spaces can improve living conditions and enhance the overall quality of life [23]. A geographical neighbourhood which is not supportive to walking, and with limited access to services and resources, poses further significant barriers for older people seeking full participation in society, with deleterious effects predisposing the development of geriatric depressive symptoms $[13,41]$. Moreover, several studies have shown that certain neighbourhood factors were consistently associated with depression [14, 35, 51, 52]. Indeed, it is found that seniors facing severe mobility impairment have a lower level of social engagement and life satisfaction [29], suggesting that poor accessibility can create environmental stressors to the elderly.

In terms of the interactions of personal and environmental resources, the prevalence of depression has been found to be affected by the number of rooms in the home, as well as housing quality [14]. A higher satisfaction with housing was associated with fewer depressive symptoms [28]. Several self-reported measures of the geographical neighbourhood, such as neighbourhood collective efficacy, neighbourhood problems, and neighbourhood quality, were also important. Generally, living in a low-income neighbourhood appears to be strongly related to poorer mental health and depression [16]. The extent, strength, and quality of social connections with each other in their neighbourhoods affect their appraisal and coping responses and, hence, is also a social determinant of health [19]. 'Non-material' aspects of life, such as support and love, are necessary for good mental health [3]. Hence, the emotional support older people receive within their local neighbourhood is particularly relevant. Studies suggest that the concentration of older people living close together could have a favourable effect on alleviating geriatric depression [35]. There is also a body of evidence showing that social capital-the degree to which older people see themselves as involved with, and are able to rely on, other members in their neighbourhood [40] — buffers against stressful life events, improves

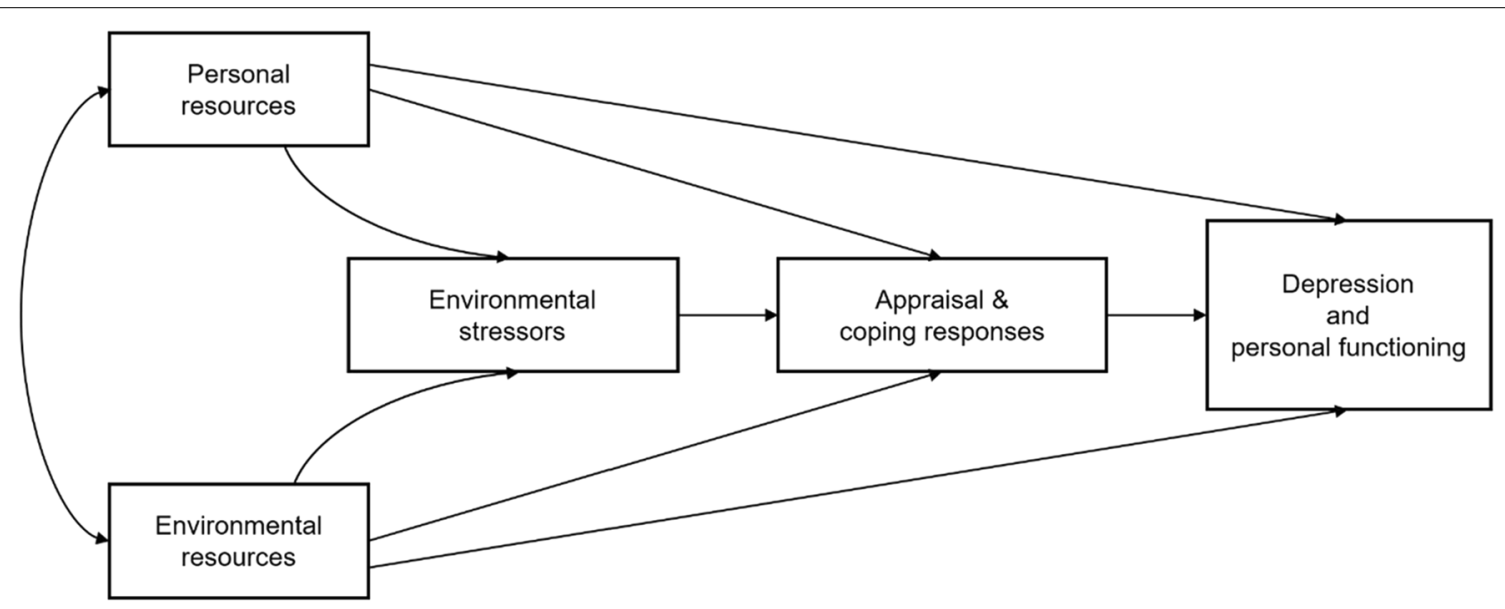

Fig. 1 An integrative framework of people-environment factors in analysing adaptive processes and depression [4] 
coping responses, and helps prevent the onset of depression [26]. Social capital has important moderating or mediating effects on depression in different populationbased cohort studies [3], including a sampled Chinese population [6].

Despite the growing interest in understanding the environmental influences on people's health, research studies have mainly focused on the West. Much is still unknown about the importance of the neighbourhood environment on depression in Asian communities, which tend to be of higher density and diversity $[9,51,52]$. The study by Chen et al. focused on the neighbourhood characteristics of elderly people living in low-income communities in Hong Kong. By assessing perceived measures using linear regression, they found that at-ease walking proximity to medical facilities was influential [9]. The work by Chen's team adds to the knowledge about particular aspects of neighbourhood environmental resources on geriatric mental health in Asian communities. Moreover, the empirical evidence in three Asian cities (i.e., Hong Kong, Singapore, and Tokyo) indicates that the home environment of the elderly, especially neighbourhood walkability, is interconnected with the formation of social capital and the elderly's physical and mental health conditions [34]. The research team of Zhang et al. [51, 52] further explored the environment-depressive symptoms associations and found that the "ultra-dense, well-connected, pedestrian-friendly, destination-rich neighbourhoods may contribute to lowering the risk of depressive symptoms in Hong Kong older adults by enabling them to frequently walk to local destinations of daily living and, thus, maintain their independence and bond with the community" (Zhang et al. [52]: 96). Yet, exposure to extreme levels of public transport density and associated traffic volumes may create environmental stress and increase the risk of depressive symptoms [51].

Our study aims to complement and expand the existing literature by revisiting the definition of geographical neighbourhood, incorporating both objective and subjective measures of the neighbourhood, and by extending the study area to include both Hong Kong and Singapore-two cosmopolitan Asian cities-to elucidate effective strategies ahead. It is increasingly recognized that a combination of objective and subjective measures is necessary in understanding complex public health issues [22]. By employing structural equation modelling (SEM), we examine the possible mechanisms by which depressive symptoms may be related to the neighbourhood environment in which a person resides. Hong Kong and Singapore were selected in view of their many common characteristics, physically, economically, historically, and culturally. The contrast between the two societies may well serve to inform the debate on geriatric depression, which thus far has centred primarily on Western countries.

\section{Methodology \\ Sample population and study area}

This cross-sectional study involved community-dwelling senior citizens in Hong Kong and Singapore with support from local senior citizen community centres. Due to the lack of comprehensive sampling frames, convenience sampling was used. The survey altogether included 228 seniors in Hong Kong and 250 seniors in Singapore. Written consent was obtained from all participants before the interviews, which were conducted during May and June, 2013. More details about the survey design and sample recruitment are provided in Loo et al. [33]. The neighbourhoods in Hong Kong were selected based on the comprehensive list of senior community centres from the Social Welfare Department. All senior community centres were approached for participation. Upon initial agreement to participate, site visits were done to establish the suitability of the venue, time schedule, and membership size. The samples eventually came from four neighbourhoods in Sai Ying Pung on Hong Kong island, Hung Hom and Lai Chi Kok in Kowloon, and Tai Po in the New Territories. In Singapore, the seniors were recruited at an established training and research centre in the Jurong town area. This is the same large scale satellite housing development that is seen across the country; each is self-contained with public housing units, a town centre, amenities for shopping, employment in industrial estates, schools, medical care and recreational facilities. Based on the respondents' home locations, the three neighbourhoods are Jurong West Central, Boon Lay, and Hong Kah. Major road networks generally do not run through these townships which are however linked to these major highways. In both cities, our target population were healthy community-dwelling elderly who are not experiencing any acute life event known to the elderly community or research training staff.

Based on the samples, further inclusion criteria for the study population were people who (a) were aged 65 years or older in the study period; (b) scored $20 / 22$ or above in MMSE during the screening of cognitive abilities (see below); and (c) completeness of the questionnaires. This resulted in a total sample size of 347, with 173 and 174 elderly people in Hong Kong and Singapore respectively. More specifically, 26, 47, and 58 samples were excluded because of the above three inclusion criteria, respectively.

\section{Demographic data collection}

The gathered demographic and socioeconomic information of the respondents included: gender, age, educational level, living arrangements and accommodation, 
household car-ownership, and number of chronic diseases. The body mass index (BMI) for all respondentsas an indicator of general obesity-was also recorded.

\section{Assessments}

The Mini-Mental State Examination (MMSE) was used to measure cognitive impairment. This 30-point questionnaire tests various cognitive functions, including orientation to time and place, repetition, verbal recall, attention and calculation, language, and visual construction. The total test score ranges from 0 (impaired) to 30 (normal). Cut-off points for cognitive impairment of 22 for those who are literate and 20 for those who are illiterate were based on a previously validated study in the Chinese population which yielded a sensitivity of $83.87 \%$ and a specificity of $84.48 \%$ [49].

The 15-item Geriatric Depression Scale (GDS-15) was used to assess depression. Each item was scored on a dichotomous 'Yes' or 'No' response, and the scale was the sum of the recoded 15 items, ranging from 0 to 15. Higher scores on the GDS indicate higher levels of depressive symptoms. The prevalence of depression was indicated by a cut-off point of 8 [11]. For Cantonese speakers, the validated Chinese version of the GDS was used. Cronbach's alpha in the present sample is 0.83 , suggesting a reasonably reliable measure for further analysis.

Health-related variables were obtained using the SF36v2 Health Survey-a multipurpose, short-form health survey. It has 36 questions that yield an eight-scale profile of functional health and well-being, two psychometrically based physical and mental health summary measures, and a preference-based health utility index. The physical component score (PSC) and mental component score (MCS) are two variables used to reflect the general physical and mental health status respectively [33]. Both have a range of 0 to 100 with higher scores suggesting better health [46]. For easy reference, they can be interpreted as summary physical and mental health scores, respectively.

The International Physical Activity QuestionnaireShort Form (IPAQ-SF) was used, which measures health-related participation in physical activity (PA) in populations. The specific types of activity assessed are: walking, moderate-intensity activities, and vigorous intensity activities. Frequency (measured in days per week) and duration (time per day) are collected separately for each specific type of activity. Items are structured to provide separate scores for walking, moderate-intensity, and vigorous-intensity activity, as well as a combined total score to describe overall level of activity. Volume of activity is computed by weighting each type of activity by its energy requirements as defined in the Metabolic Equivalent of Tasks (METs), which are multiples of the resting metabolic rate. Multiplying the MET score with the number of minutes the activity is performed yields a score in MET-minutes. MET-minutes per week were used to classify older people into three categories: low, moderate, and high level of physical activity [21].

\section{Geographical neighbourhood variables}

Indicators of two sets of geographical neighbourhood variables, based on immediacy and breadth of impact, were constructed. Level-1 local factors or individualspecific immediate neighbourhood factors refer to parameters at a micro scale of influence, while those at a meso-level are termed level-2 local factors or wider shared neighbourhood factors. This study advocates the concept of people-based neighbourhoods, which are identified based on the actual activity space or spacetime movements of individuals [33] rather than districtbased neighbourhoods, which are fixed for administrative purposes. To achieve this, the home addresses of all participants were geocoded using a geographic information system (GIS). To analyse level-1 local factors, a 500-m network buffer was drawn around each participant's residential location using network distance to reflect walkable distance for the participants [7]. Neighbourhood factors were gaugfed on a pro-rata basis.

The first dimension of level-1 local factors captures the spatial distribution of opportunities near their homes as this plays a key role in defining social in/exclusion [41]. By calculating the shortest physical distance to key facilities around the participants' homes [50], this study considers the network distance to places such as the nearest medical facility (DIS1) or entrance to an open space (DIS2). Network distance here refers to the length of walking path on the pedestrian network, which is more realistic than the shortest direct line/Euclidian distance, between two places [32]. The second dimension of level-1 local factors captures various key principles in urban planning, including connectivity $(\mathrm{CON})$ and the $3 \mathrm{D}$, that is, density (DEN), diversity (DIV), and design (DES) [8]. $\mathrm{CON}$ is measured by the road junction density within the buffer area. For DEN, this study considers overall and elderly population density, where higher density implies an advantage of having more population to support a vibrant neighbourhood. DIV, refers to the mix of land use in contributing to a balanced development. It is calculated using the Simpson's Diversity Index [43] as an entropy measure by considering five categories of land use: residential, commercial, institutional, recreational, and others [32]. Finally, good DES relates to the attractiveness of the neighbourhood environment. It includes connectivity as expressed in the number of intersections per square kilometre (DES1), the percentage of open space in one's neighbourhood (DES2a), and the area $\left(\mathrm{km}^{2}\right)$ of parks contained within the network buffer. 
The third dimension relates to one's perception of how walkable is the neighbourhood. Eighteen subjective walkability variables under the dimensions of comfort, convenience and safety were gathered from the face-to-face questionnaire survey. These variables are summarized in Table 1 . The different parameters of the neighbourhood walking environment were rated on a Likert scale from one to five, ranging from very poor to very good condition. In addition, participants were also asked to rate the overall walkability of their neighbourhood subjectively on a scale of 0 to 100 .

To measure social capital, four statements were used. Three statements required a response on a five-point Likert scale from strongly disagree to strongly agree: "People in my neighbourhood get along with each other well"; "People are willing to help each other"; and "Living in this neighbourhood gives me a sense of community". The fourth statement, "Do you have someone to accompany you outside?", required a yes or no answer.

The above neighbourhood factors are individual-specific. This poses a challenge to the operationalization of the shared space, which can enhance the relationship between people, places, and the surrounding traffic $[17,18]$. Spatial aggregation was then performed in the study by overlapping individual neighbourhood buffers with common boundaries and within a walkable distance roughly double the immediate neighbourhood buffer of $500 \mathrm{~m}$ (that is, a radius of roughly $1 \mathrm{~km}$ ). Level- 2 local factors or the wider shared community factors capture the general or average condition of people living there. The first dimension of level-2 local factors captures the average or mean value of all parameters included in the level-1 local factors. The second dimension covers some additional demographic variables. These include the percentage of oldest-old of 85 years or above and females. The third dimension includes an objective walkability assessment, which is a composite score of the walking conditions of the neighbourhood based on the research protocols developed by Loo and Lam [32]. There are altogether twelve variables, covering the pavement (pavement surface, shelter, pedestrian guardrail, directional signs, road works and street furniture) and crossing facilities (dropped kerb, audible pedestrian signals, number of vehicular lanes, refuge island for wide roads, traffic light cycle, and crossing time).

\section{Research framework}

SEM is used to establish relationship strength and direction among the different individual and neighbourhood environment parameters in the occurrence of depressive symptoms among the elderly. It is also used to test the mediating relationship, elucidating the potential pathways in which neighbourhood factors and individual attributes affect depression in the elderly. SEM is particularly suitable to this research because it allows for the building of complex models (including relationships among observed and latent-variables) and corrects for measurement errors, thereby allowing for a more accurate test of the mediational effect [25]. Based on our theoretical framework, we have selected different factors related to personal resources and environmental resources as exogenous variables The latter are further classified as individual-specific immediate neighbourhood characteristics (hereafter referred to as level 1 local factors) and wider shared neighbourhood characteristics (hereafter referred to as level 2 local factors). Depressive

\section{Table 1 Subjective ratings of the walking environment in a neighbourhood}

\begin{tabular}{|c|c|}
\hline Dimension & Variables \\
\hline Comfort & $\begin{array}{l}\text { There are too many people on the streets and it makes it difficult or unpleasant to walk } \\
\text { There are covered sidewalks and/or indoor area (e.g. shopping centre) to walk } \\
\text { The degree of air pollution is high in my neighbourhood } \\
\text { There are enough beautification and enhanced greenery along the streets in my neighbourhood } \\
\text { There are many attractive sceneries to look at while walking in my neighbourhood } \\
\text { Streets are not clean in my neighbourhood }\end{array}$ \\
\hline Convenience & $\begin{array}{l}\text { It is easy to walk to transport stations from my home } \\
\text { Stores are within easy walking distance of my home } \\
\text { There are many alternative routes for getting from place to place in my neighbourhood } \\
\text { There are clear road signs and pedestrian signals } \\
\text { There are enough facilities to take rest (e.g. benches) } \\
\text { Pedestrian bridges or subways are well equipped with sufficient lifts or escalators }\end{array}$ \\
\hline Safety & $\begin{array}{l}\text { There is so much traffic that it makes me feel insecure to walk on the streets } \\
\text { The pedestrian green light time is too short for me to cross the road safely } \\
\text { The crime condition in my neighbourhood makes it unsafe to walk } \\
\text { Sidewalks conditions are poor (Not flat or slippery) } \\
\text { There are many slopes or stairs that make me feel insecure to walk } \\
\text { My neighbourhood streets are not well lit at night }\end{array}$ \\
\hline
\end{tabular}


symptoms were treated as endogenous. Thus, it is hypothesized that the exogenous variables interact with and mediate the development of depressive symptoms. The dependent variable in this study is the occurrence of depressive symptoms among the elderly population.

\section{Results}

The characteristics of the sample population are shown in Table 2. The overall prevalence of depression using the GDS cut-off point of 8 was $7.8 \%$ in Hong Kong and $15.6 \%$ in Singapore. It should be noted that convenience sampling was adopted in this research work. In all of the 347 older people, a large proportion (77.2\%) were female $(n=268)$. The higher percentage of females in the sample reflects a gender difference in attitudes towards involvement in community and volunteer activities in the two cities [38]. Despite this, the present multi-centre study is an effective method of recruiting sufficient number of community-based subjects. The mean age of the elderly respondents was 73.82 years $(\mathrm{SD}=6.04)$, ranging from 65 to 95 years. $4.9 \%$ of the samples belong to the oldestold age, who were aged 85 or above during the study year. Around $23.3 \%(n=81)$ of respondents lived alone and $77.5 \%$ were part of non-car owning households $(n=269)$. About $70 \%$ of the respondents $(n=243)$ only received primary education or lower level. Following the

Table 2 Descriptive analysis of the sample

\begin{tabular}{|c|c|c|c|c|c|c|}
\hline \multirow[t]{2}{*}{ Parameters } & \multicolumn{2}{|c|}{ Hong Kong } & \multicolumn{2}{|c|}{ Singapore } & \multicolumn{2}{|c|}{ Total } \\
\hline & $\mathbf{N}$ & $\%$ & $\mathbf{N}$ & $\%$ & $\mathbf{N}$ & $\%$ \\
\hline \multicolumn{7}{|l|}{ Gender } \\
\hline Male & 20 & 11.6 & 59 & 33.9 & 79 & 22.8 \\
\hline Female & 153 & 88.4 & 115 & 66.1 & 268 & 77.2 \\
\hline \multicolumn{7}{|l|}{ Age group } \\
\hline $65-70$ & 30 & 17.3 & 87 & 50.0 & 117 & 33.7 \\
\hline $71-75$ & 41 & 23.7 & 56 & 32.2 & 97 & 28.0 \\
\hline $76-80$ & 62 & 35.8 & 26 & 14.9 & 88 & 25.4 \\
\hline $81-85$ & 27 & 15.6 & 3 & 1.7 & 30 & 8.6 \\
\hline Above 85 & 13 & 7.5 & 2 & 1.1 & 15 & 4.3 \\
\hline Living alone & 65 & 37.6 & 16 & 9.2 & 81 & 23.3 \\
\hline Car-owning household & 9 & 5.2 & 69 & 39.7 & 78 & 22.5 \\
\hline \multicolumn{7}{|l|}{ Education level } \\
\hline Primary or lower & 136 & 78.6 & 107 & 61.5 & 243 & 70.0 \\
\hline Junior secondary & 20 & 11.6 & 34 & 19.5 & 54 & 15.6 \\
\hline Senior secondary or above & 17 & 9.8 & 33 & 19.0 & 50 & 14.4 \\
\hline \multicolumn{7}{|l|}{ BMl group } \\
\hline Underweight & 6 & 3.5 & 10 & 5.7 & 16 & 4.6 \\
\hline Normal range & 93 & 53.8 & 108 & 62.1 & 201 & 57.9 \\
\hline Overweight & 65 & 37.6 & 48 & 27.6 & 113 & 32.6 \\
\hline Obese & 9 & 5.2 & 8 & 4.6 & 17 & 4.9 \\
\hline \multicolumn{7}{|l|}{ Lifestyle } \\
\hline Inactive & 5 & 2.9 & 70 & 40.2 & 75 & 21.6 \\
\hline Minimally active & 90 & 52.0 & 92 & 52.9 & 182 & 52.4 \\
\hline Active & 78 & 45.1 & 12 & 6.9 & 90 & 25.9 \\
\hline Three or more kinds of chronic illnesses & 42 & 24.3 & 40 & 23.0 & 82 & 23.6 \\
\hline \multicolumn{7}{|l|}{ Physical health score } \\
\hline Below 50 & 36 & 20.8 & 9 & 5.2 & 45 & 13.0 \\
\hline $50-74$ & 66 & 38.2 & 87 & 50.6 & 153 & 44.3 \\
\hline 75 or above & 71 & 41.0 & 76 & 44.2 & 147 & 42.6 \\
\hline \multicolumn{7}{|l|}{ Mental health score } \\
\hline Below 50 & 13 & 7.5 & 8 & 4.6 & 21 & 6.1 \\
\hline $50-74$ & 49 & 28.3 & 61 & 35.3 & 110 & 31.8 \\
\hline 75 or above & 111 & 32.2 & 104 & 60.1 & 215 & 62.1 \\
\hline
\end{tabular}


guidelines of the World Health Organization [48], around two third of respondents were normal-weight individuals (a BMI of 18.5 to 24.99). Nevertheless, $32.6 \%$ of the elderly people in this study were overweight and $4.9 \%$ were obese. Approximately $23.6 \%$ had more than three chronic diseases, of which the top three were high blood pressure (51.8\%), knee osteoarthritis (31.1\%), and diabetes (18.6\%). Despite these constraints, many respondents in Hong Kong remained physically active $(45.1 \%)$ based on the score in MET-minutes. The share of being physically active was lower in Singapore (6.9\%). Moreover, as seen from Table 2, our subject individuals generally enjoyed reasonably good physical and mental health as community-dwelling elderly of over 65 years old. Overall, less than $20 \%$ of the respondents have their physical and mental health scores below 50 . However, as shown above, these average figures hide problems of depression in the ageing population.

Following the SEM approach, we have tested different models and the final model with accompanying path coefficients is presented in Fig. 2. Only statistically significant variables are kept. Examining the factors that characterize older people's neighbourhoods may help provide evidence as to the extent to which neighbourhood factors are related to the development of depressive symptoms. Firstly, the model shows that better physical conditions, as reflected by a higher physical health score, are linked to a lower risk in depressive symptoms (coefficient $=-0.01$, $\mathrm{p}<0.05$ ). While overweight or obese elderly people

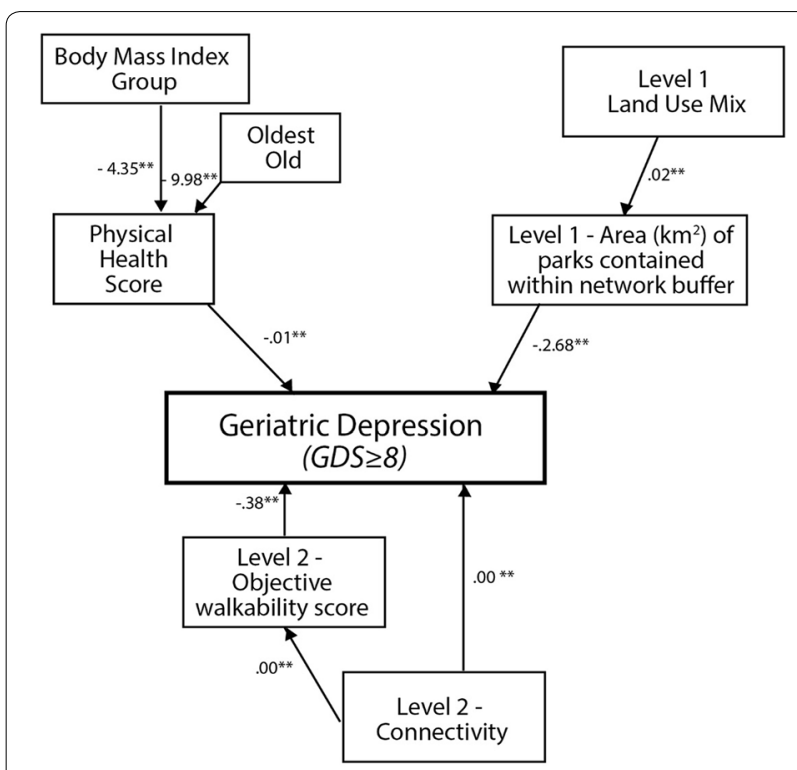

Significance level: ** $p<0.01 ;{ }^{*} p<0.05$.

Fig. 2 Path diagram showing the relationships between individual and neighbourhood factors (coefficient $=-4.35 ; \mathrm{p}<0.05$ ), and the oldest-old (coefficient $=-9.98 ; \mathrm{p}<0.05$ ), are less likely to maintain a good physical health status. Among the immediate neighbourhood factors (Level 1), abundant park area (coefficient $=-2.68 ; \mathrm{p}<0.05)$ help to counteract depression. The park area is, in turn, affected by land use mix (coefficient $=0.02 ; \mathrm{p}<0.05$ ), suggesting that higher land use mix is positively associated with the park area. Among the wider shared neighbourhood factors (Level 2), good objective walkability score (coefficient $=-0.38 ; \mathrm{p}<0.05$ ) is negatively associated with depressive. In addition, the good objective walkability score is positively associated with connectivity (coefficient $=-0.00 ; \mathrm{p}<0.05$ ), though the variable itself is also directly associated with geriatric depression (coefficient $=-0.00 ; \mathrm{p}<0.05$ ). Generally, this suggests and reconfirms that higher road junction density (CON or connectivity) can create smaller street blocks to encourage walking but heavy vehicular traffic may also create environmental stress.

In addition, some other variables that we have tested are also generating results that are worth mentioning, though their statistical significance levels are not below 0.05 to be reported in Fig. 2. They are subjective variables. Two are individual-specific immediate neighbourhood (level-1) factors, that is, the subjective sense of community (coefficient $=-0.02$ ) and subjective perception of poor neighbourhood air quality (coefficient $=0.01$ ), and two are wider shared neighbourhood (level-2) factors, that is, having companions to walk together (coefficient $=-0.89$ ), and getting along well with neighbours (coefficient $=-0.30$ ). We believe that the limited sample size may be a contributory factor and these subjective neighbourhood factors are worth closer examination in future larger-scale research.

\section{Discussion}

Referring to the integrative framework of Billings and Moos [4], our work indicates that personal resources, such as physical health and body weight, are closely linked to the elderly's mental health. This is contrary to previous studies which have showed that there is either no apparent association observed between BMI and depressive symptoms [27], or that there is even a negative relationship [30]. This present study suggests that maintaining a healthy weight allows one to be in better physical health condition and, in turn, has better mental health. Fitness programmes for the elderly, such as outdoor walking activities, could be further promoted at a community-wide level, in order to keep them active and physically fit.

In terms of environmental resources, we found that having larger areas of parks near one's home is linked to fewer depressive symptoms. In Hong Kong and 
Singapore, parks represent open green space and leisure facilities such as playground, basketball courts and sports complex. One can conceptualize the impact in two ways. First, parks has been found to be important to people whether or not they use it often and actively [36]. Parks seen at a distance from one's home can also be viewed as an environmental resource of importance to public mental health [1]. Second, being able to be directly immersed in the leisure environment provides opportunities for morning exercises, after-dinner walks, and social contacts, which are beneficial to older people's health and well-being. Particularly in high-rise compact urban areas such as Hong Kong and Singapore, daily contact with the natural environment can have measurable mental benefits [10]. An earlier study indicated that once access is considered, size is more important than attractiveness in determining use [15]. This echoes the study result where the size of parks near to one's home matters.

Another significant and powerful environmental resource that helps to reduce the risk of geriatric depression in Hong Kong and Singapore is promoting walkability within one's neighbourhood. Walking is a popular, inexpensive, and convenient activity for older adults that is vital to maintaining their physical and mental health [32, 45]. A supportive neighbourhood environment helps promote walking as a mode of active transport that can be incorporated into the daily routine of older people, which often involves making medical trips [31]. By adopting an objectively measured micro-scale walkability assessment to evaluate different dimensions of the walking environment, this study found that promoting a more walkingfriendly neighbourhood can be a core component of the public health policy. The composite score suggests that pavement width, surface condition, availability of street seats, ease of finding assistance, pedestrian crossing width, and whether or not the overpasses/underpasses are equipped with lifts, are important contributing factors in a walkable environment that decreases the risks of geriatric depression. A previous study discovered that, in a Western context, a more walking-friendly neighborhood decreases the risk among the elderly of becoming depressed [2].

\section{Conclusion}

This study offers an integrated analysis of neighbourhood effects on geriatric depression; and provides empirical evidence suggesting that an older person's neighbourhood of residence contributes to geriatric mental health. A walkable environment with abundant parks are favourable environmental settings for older people in Hong Kong and Singapore. To date, knowledge is very limited regarding the variability of neighbourhood effects among Asian cities on a person's mental health. By exploring the effects of neighbourhood environment on geriatric depression, the results have paved the way for further studies on intercity or regional comparison of mental health in an ageing population globally. The present study also adds to the diversity of available population-based literature on geriatric depression in Asia. Theoretically, the study stresses that the concept of geographical neighbourhood that goes beyond the immediate nearby areas but also the wider community. The first set of neighbourhood factors, which we referred to as level-1 local factors, can be individualspecific. It reflects one's perceptions of his/her neighbourhood. The second set of neighbourhood characteristics, referred to as level-2 local factors, is related to certain general or common conditions that people share in a local area. The advantages at the different levels that the assessment revealed can provide a more robust understanding of the issues, while also underlining the challenges that exist in defining neighbourhoods; which is rather a complex concept to define and measure.

This study is not without its limitations. First, the sampling strategy could be improved by trying to reach community-dwelling elderly directly. Given the difficulties of getting a better sampling frame, convenience sampling was used. Moreover, the sample size is limited. As SEM with multiple explanatory factors perform better with bigger samples [29], increasing the sample size will allow us to model more variables and further explore the complex relationships of subjective variables with depression. Furthermore, respondents in Hong Kong and Singapore are pooled for the analysis, differences among communities and between cities are not examined in this study. Further research should address variations at different spatial scales. Second, the research investigated the presence of clinically relevant levels of depressive symptoms using GDS rather than the clinical diagnosis of depression. However, GDS is one of the most commonly used depressive symptoms measures in geriatric studies and has been shown to have good reliability for clinical diagnosis. Third, the dependent variable for the analytical data analysis is binary in nature. This can lead to concerns over the prevalence and incidence of depressive symptoms, and reduce the predictive power of the model. Though the binary approach adopted is sufficient in addressing the manifold question at hand, it does place constraints on a better understanding of geriatric depression. Fourth, the cross-sectional design adopted poses a number of challenges. It is not possible to unequivocally determine the direction of causation using the present cross-sectional data. For instance, depression at any given time might be influenced by current neighbourhood features. On the other hand, it can also be the result of cumulative exposure to neighbourhood features over many years. The effects of the neighbourhood environment on the 
development of depression is likely to have a time lag. This calls for further, preferably longitudinal, research to adequately explain the causal pathways by which a neighbourhood might affect health. Very often, depression is best viewed across a longitudinal time series which transects through the invasion history of depression into a person's mind [39]. Besides, as our research did not collect the elderly residential history and decision-making information, we cannot conduct meaningful analysis about the residential self-selection issue. We recognize that focused research may further explore its relationship with depression.

On the way forward, the findings of this research have political implications for both Hong Kong and Singapore, as they are faced with rapidly ageing populations and socio-cultural changes. Overall, the high prevalence rates of geriatric depression in many ageing societies has led to public concerns. So far, the ageing policy has put much attention on the financial, healthcare and housing aspects. Nonetheless, this paper finds that the geographical neighbourhood in which an older person lives has a significant impact on his/her mental health, even after accounting for individual-level determinants. Even though depression is an individually experienced phenomenon, external factors including the neighbourhood environment are linked to its development. The development, evaluation, and dissemination of a new generation of programmes and structural interventions, especially on how to improve the neighbourhood environment, can be targeted towards improving the mental health of this population. Above all, understanding depression requires an analysis of the complex web of variables that gave rise to these specific individual circumstances. The expanding body of research on the effect of the neighbourhood environment on depression holds great promise, not only for achieving a more complete aetiological picture of the conditions, but also for delineating ways to understand and promote health and well-being in an ageing population. The findings of this study suggest that psychological health and the environment require greater age-specific policies if the elderly are to age well and successfully with good quality of life and life satisfaction.

\section{Acknowledgements}

Not applicable.

\section{Authors' contributions}

WWYL led the research fieldwork, conducted the data analysis, and drafted this paper. BPYL conceptualized the project, obtained the research funding, guided the data analysis, and edited the manuscript. RM obtained the research funding, led the research fieldwork in Singapore, and guided the data analysis. All authors read and approved the final manuscript.

\section{Authors' information}

Dr Winnie W. Y. Lam obtained her PhD degree at the Department of Geography of the University of Hong Kong. Her research interests are socially sustainable transport, especially in relation to the transport disadvantaged such as the elderly, women and children.
Professor Becky P. Y. Loo is Professor and Head of Department of Geography Head of Department at the University of Hong Kong. She is a Fellow of the Academy of Social Sciences, UK. She is also the Director of the Institute of Transport Studies, and Founding Co-Director of the Joint Laboratory on Future Cities. Her core research interests are transportation, smart technologies and cities. In particular, she is interested in applying spatial analysis, surveys and statistical methods in analysing pertinent issues related to the relationships of the environment and the well-being of people living in cities.

Dr Rathi Mahendran is Associate Professor \& Senior Consultant Psychiatrist, Department of Psychological Medicine, Yong Loo Lin School of Medicine, National University of Singapore. She graduated from the National University of Singapore in 1980 and completed the Specialist Training in Psychiatry in 1998. She subsequently trained in the UK and the US under Health Manpower Development Plan programmes in 1989 and 1996 respectively. She was awarded Master of Medical Education from the University of Dundee (UK) in 2013. Her interests are in psychopharmacology and medical education. She also provides psychosocial care for ambulatory patients at the National University Cancer Institute, Singapore (NCIS). Some of Dr Rathi's notable achievements include the Distinguished Psychiatrist Award by Singapore Psychiatric Association, awarded in 2016.

\section{Funding}

This research project was funded by the National University of Singapore (NUS) Initiative to Improve Health in Asia (NIHA) Research Grant on "Promoting Active Transportation for the Elderly: A Comparative Study of Three Asian Cities" (NIHA-2011-1-010). It was also partially funded by the Sixth Government Matching Grant Scheme on "Health and Active Ageing: What is the Role of the Urban Environment" of the Hong Kong Special Administrative Region Government and the 27th Round Post-doctoral Fellowship/Research Assistant Professorship Scheme on "Healthy Aging and Sustainable Transport" of the University of Hong Kong.

Availability of data and materials

The datasets collected in the current study are available from the corresponding author on reasonable requests.

\section{Ethics approval and consent to participate}

All participants in this survey have signed consent to participate. The ethical approvals have been obtained from the University of Hong Kong (EA280812) and the National University of Singapore (NHG DSRB Ref: 2013/00047).

\section{Consent for publication}

All authors agreed to publish the paper in the International Journal of Health Geographics.

\section{Competing interests}

There are no competing interests to declare.

\section{Author details}

${ }^{1}$ Department of Geography, The University of Hong Kong, Pokfulam Road, Hong Kong, China. ${ }^{2}$ Department of Psychological Medicine, National University of Singapore, Singapore, Singapore.

Received: 19 June 2020 Accepted: 5 October 2020

Published online: 13 November 2020

\section{References}

1. Adams LW. History of urban wildlife conservation. Urban Wildlife conservation: Springer; 2014. p. 11-31.

2. Berke EM, Gottlieb LM, Moudon AV, Larson EB. Protective association between neighborhood walkability and depression in older men. J Am Geriatr Soc. 2007:55(4):526-33.

3. Berkman LF, Glass T. Social integration, social networks, social support, and health. Social Epidemiol. 2000;1:137-73.

4. Billings AG, Moos RH. Psychosocial theory and research on depression: an integrative framework and review. Clinical Psychol Rev. 1982;2:213-37. 
5. Blazer DG. Depression in late life: review and commentary. J Gerontol Series A Biol Sci Med Sci. 2003;58(3):M249-65.

6. Cao W, Li L, Zhou X, Zhou C. Social capital and depression: evidence from urban elderly in China. Aging Mental Health. 2015;19(5):418-29.

7. Cervero R. Transit-oriented development's ridership bonus: a product of self-selection and public policies. Environ Plan A. 2007;39(9):2068-85.

8. Cervero R, Kockelman K. Travel demand and the 3Ds: Density, diversity, and design. Transportation Res Part D Transport Environ. 1997:2(3):199-219.

9. Chen Y-Y, Wong GH, Lum TY, Lou VW, Ho AH, Luo H, et al. Neighborhood support network, perceived proximity to community facilities and depressive symptoms among low socioeconomic status Chinese elders. Aging Mental Health. 2016;20(4):423-31.

10. Chiesura A. The role of urban parks for the sustainable city. Landscape Urban Plan. 2004;68(1):129-38.

11. Chiu H, Lee H, Wing Y, Kwong P, Leung C, Chung D. Reliability, validity, and structure of the Chinese Geriatric Depression Scale in a Hong Kong context: a preliminary report. Singapore Med J. 1994;35:477.

12. Fiske A, Wetherell JL, Gatz M. Depression in older adults. Ann Rev Clin Psychol. 2009:5:363-89.

13. Fonda SJ, Wallace RB, Herzog AR. Changes in driving patterns and worsening depressive symptoms among older adults. J Gerontol Series B Psychol Sci Social Sci. 2001;56(6):S343-51.

14. Garin N, Olaya B, Miret M, Ayuso-Mateos JL, Power M, Bucciarelli P, et al. Built environment and elderly population health: a comprehensive literature review. Clin Pract Epidemiol Mental Health. 2014;10:103.

15. Giles-Corti B, Broomhall MH, Knuiman M, Collins C, Douglas K, Ng K, et al. Increasing walking: how important is distance to, attractiveness, and size of public open space? Am J Prev Med. 2005;28(2):169-76.

16. Glass TA, Balfour JL. Neighborhoods, aging, and functional limitations. Neighborhoods Health. 2003:1:303-34

17. Hamilton-Baillie B. Towards shared space. Urban Design Int. 2008;13(2):130-8

18. Hammond V, Musselwhite $C$. The attitudes, perceptions and concerns of pedestrians and vulnerable road users to shared space: a case study from the UK. J Urban Design. 2013;18(1):78-97.

19. Hernandez LM, Blazer DG. Genes, behavior, and the social environment: moving beyond the nature/nurture debate. Washington (DC): National Academies Press; 2006.

20. Information Services Department. LCQ7: Mental health services. 2012. https://www.info.gov.hk/gia/general/201203/28/P201203280399.htm

21. IPAQ Research Committee. Guidelines for data processing and analysis of the International Physical Activity Questionnaire (IPAQ)-short and long forms. https://www.ipaq.ki.se. 2005.

22. Ivaldi E, Bonatti G, Soliani R. Objective and subjective health: an analysis of inequality for the European Union. Soc Indicator Res. 2018;138(3):1279-95.

23. Jackson LE. The relationship of urban design to human health and condition. Landscape Urban Plan. 2003;64(4):191-200.

24. Julien D, Richard L, Gauvin L, Kestens Y. Neighborhood characteristics and depressive mood among older adults: an integrative review. Int Psychogeriatr. 2012;24(8):1207-25.

25. Kaplan D. Structural equation modeling: foundations and extensions. 2nd ed. Thousand Oaks, California: Sage Publications; 2009.

26. Kawachi I, Berkman LF. Social ties and mental health. J Urban Health 2001;78(3):458-67.

27. Kuriyama S, Koizumi Y, Matsuda-Ohmori K, Seki T, Shimazu T, Hozawa A, et al. Obesity and depressive symptoms in elderly Japanese: the Tsurugaya Project. J Psychosom Res. 2006;60(3):229-35.

28. Lai DW, Guo A. Gender differences in depressive symptoms of aging Chinese in urban Jiangsu in China. Clin Gerontol. 2011;34(3):190-206.

29. Li L, Loo BP. Mobility impairment, social engagement, and life satisfaction among the older population in China: a structural equation modeling analysis. Qual Life Res. 2017:26(5):1273-82.

30. Bin Li Z, Yin Ho S, Man Chan W, Sang Ho K, Pik Li M, Leung GM, et al. Obesity and depressive symptoms in Chinese elderly. Int I Geriatric Psychiatry. 2004;19(1):68-74

31. Loo BPY, Lam WWY. Geographic accessibility around health care facilities for elderly residents in Hong Kong: a microscale walkability assessment. Environ Planning B Planning Design. 2012;39(4):629-46.
32. Loo BPY, Lam WWY. A multilevel investigation of differential individual mobility of working couples with children: a case study of Hong Kong. Transportmetrica A: Transport Sci. 2013;9(7):629-52.

33. Loo BPY, Lam WWY, Mahendran R, Katagiri K. How is the neighborhood environment related to the health of seniors living in Hong Kong, Singapore, and Tokyo? Some insights for promoting aging in place. Ann Am Assoc Geographers. 2017;107(4):812-28.

34. Loo BPY, Mahendran R, Katagiri K, Lam WWY. Walking, neighbourhood environment and quality of life among older people. Curr Opin Environ Sustainability. 2017;25:8-13.

35. Mair CF, Roux AVD, Galea S. Are neighbourhood characteristics associated with depressive symptoms? a review of evidence. J Epidemiol Community Health. 2008;62(11):940-6.

36. Maller C, Townsend M, Pryor A, Brown P, St LL. Healthy nature healthy people:'contact with nature' as an upstream health promotion intervention for populations. Health Promotion Int. 2006;21(1):45-54.

37. Mehta KK. The shaping of social policies in relation to demographic ageing in East Asia. Handbook on East Asian Social Policy: Edward Elgar Publishing; 2013.

38. Mjelde-Mossey LA, Chi I. Gender differences in expectations predictive of volunteer experience among older Chinese professionals in Hong Kong. J Ethnic Cultural Diversity Social Work. 2005;13(4):47-64.

39. Parker GB. Commentary on diagnosing major depressive disorder: ask less that we embrace major depression and ask more what the concept does for us. J Nerv Ment Dis. 2006;194(3):155-7.

40. Pollack CE, Von dem Knesebeck O. Social capital and health among the aged: comparisons between the United States and Germany. Health Place. 2004;10(4):383-91.

41. Ribeiro V, Remoaldo P, Gutiérrez J. Mapping transport disadvantages of elderly people in relation to access to bus stops: contribution of geographic information systems. Accessibility and Spatial Interaction: Edward Elgar Publishing; 2014. p. 156-74.

42. Schlossberg M, Greene J, Phillips PP, Johnson B, Parker B. School trips: effects of urban form and distance on travel mode. J Am Planning Assoc. 2006;72(3):337-46.

43. Simpson EH. Measurement of diversity. Nature. 1949;163:688.

44. Subramaniam M, Abdin E, Sambasivam R, Vaingankar JA, Picco L, Pang $S$, et al. Prevalence of depression among older adults-results from the well-being of the Singapore elderly study. Ann Acad Med Singapore. 2016;45(4):123-33.

45. Warburton DE, Nicol CW, Bredin SS. Health benefits of physical activity: the evidence. CMAJ. 2006;174(6):801-9.

46. Ware JE, Kosinski M, Bjorner JB, Turner-Bowker DM, Gandek B, Maruish ME. User's manual for the SF-36v2 Health Survey. Quality Metric Incorporated; 2008.

47. Weich S, Blanchard M, Prince M, Burton E, Erens B, Sproston K. Mental health and the built environment: cross-sectional survey of individual and contextual risk factors for depression. British J Psychiatry. 2002;180(5):428-33.

48. World Health Organization. BMI classification. https://apps.who.int/ bmi/index.jsp?introPage =intro_3.html. 2006.

49. Xu G, Meyer JS, Huang Y, Du F, Chowdhury M, Quach M. Adapting Mini-Mental State Examination for dementia screening among illiterate or minimally educated elderly Chinese. Int J Geriatric Psychiatry. 2003:18(7):609-16

50. Yen IH, Michael YL, Perdue L. Neighborhood environment in studies of health of older adults: a systematic review. Am J Prev Med. 2009;37(5):455-63.

51. Zhang CJ, Barnett A, Sit CH, Lai PC, Johnston JM, Lee RS, Cerin E. Cross-sectional associations of objectively assessed neighbourhood attributes with depressive symptoms in older adults of an ultradense urban environment: the Hong Kong ALECS study. BMJ Open. 2018;8(3):e020480.

52. Zhang CJ, Barnett A, Sit CH, Lai PC, Johnston JM, Lee RS, Cerin E. To what extent does physical activity explain the associations between neighborhood environment and depressive symptoms in older adults living in an Asian metropolis? Mental Health Physical Activity. 2019:16:96-104.

\section{Publisher's Note}

Springer Nature remains neutral with regard to jurisdictional claims in published maps and institutional affiliations. 